\title{
Erscheinungsfreiheit als Therapieziel
}

\section{Die Mehrzahl der Patienten ist unzufrieden mit der derzeitigen Behandlung.}

In Österreich sind geschätzte 250.000 Personen von Psoriasis betroffen. „In den letzten Jahren hat es eine Revolution der verfügbaren Therapieoptionen für Psoriasis gegeben und viele weitere sind derzeit in klinischer Entwicklung oder ab sofort für Patienten verfügbar. Für immer mehr Patienten ist fast erscheinungsfreie oder sogar völlig erscheinungsfreie Haut ein realistisches Therapieziel. Doch der Einsatz der verfügbaren Therapie-Optionen entspricht noch immer in vielen Fällen nicht den Leitlinien - Unterversorgung und Therapieverzögerungen sind häufig", beschrieb Prof. Dr. Kristian Reich, Leiter des Dermatologikums in Hamburg die aktuelle Situation.

\section{Umfrage-Ergebnisse}

Eine im Auftrag von Novartis durchgeführte Umfrage zeigt, dass das Bewusstsein dafür, dass Psoriasis keine reine Hauterkrankung ist, auch unter Betroffenen sehr gering ausgeprägt ist, wie Dr. Wolfgang Bonitz, Medical Director von Novartis Pharma darlegte: „Knapp 60 Prozent der befragten Psoriasis-Patienten stimmten der Aussage zu, dass die Erkrankung „unangenehm und lästig, aber im Prinzip harmlos" ist, nur 25 Prozent wussten, dass Schuppenflechte oft auch mit Gelenkserkrankungen verbunden ist, nicht einmal $20 \%$, dass Psoriasis häufig auch mit anderen Begleiterkrankungen wie Diabetes oder Herz-Kreislauf-Erkrankungen, verbunden ist." Auffällig an den Ergebnissen der Umfrage ist auch, dass viele der Psoriasis-Patienten angaben, derzeit keinerlei Therapie zu erhalten - und von denen, die aktuell eine Therapie bekommen, lediglich gut ein Drittel angab, mit deren Erfolg zufrieden zu sein, nur $13 \%$ davon sehr zufrieden.

\section{Versorgungssituation in Österreich} Ein Grund dafür dürfte die verbesserungswürdige Versorgungssituation in Österreich sein, wie Doz. Dr. Paul-Gunther Sator, Leiter der Ambulanz für Psoriasis am Krankenhaus Hietzing in Wien, darlegte: „Die Ergebnisse des ,European Dermatology Health Care Survey 2013'zeigen, dass Österreich zwar einen Spitzenplatz im europäischen Vergleich innehat hinsichtlich der Anzahl an Dermatologen pro Einwohner, gleichzeitig aber nur rund $30 \%$ davon angaben, auch Biologika für die Therapie von Schuppenflechte zu verordnen. Damit liegt Österreich zwar immer noch über dem europäischen Durchschnitt, aber es zeigt sich doch, dass der Zugang zu innovativen Therapieoptionen nicht zuletzt auch stark von der Wahl des Arztes abhängt. Hinzu kommt ein starkes Stadt-Land-Gefälle was den Zugang zu innovativen Therapien betrifft - in den Städten, insbesondere in den spezialisierten Psoriasis-Ambulanzen, ist der Zugang gut, aber im ländlichen Bereich, wo die Dichte an spezialisierten Dermatologen generell sehr viel geringer ist, besteht oft noch Aufholbedarf."

Und noch ein zweiter Aspekt ist Sator besonders wichtig: „Häufig beobachte ich, dass noch immer Angst vor innovativen Therapieoptionen, insbesondere Biologika, herrscht. Hier ist ebenfalls Aufklärungsbedarf gegeben, denn viele Ängste sind heute längst überholt, die verfügbaren Therapieoptionen wirken nicht nur immer effektiver, sondern auch immer ziel- gerichteter und setzen damit immer punktgenauer an jenen Schaltstellen des Immunsystems an, die eine Schlüsselrolle für die Entstehung von Schuppenflechte und ihre Begleiterkrankungen spielen."

\section{Ziel der Behandlung}

„Weitgehende Erscheinungsfreiheit wird künftig für viele Betroffene ein realistisches Therapieziel sein. Für die Patienten bedeutet dieses Ausmaß der Besserung, dass der Betroffene nicht mehr sieht, dass er Schuppenflechte hat." Und doch herrscht auf dem Weg dorthin noch viel Handlungsbedarf, wie Reich betont: „Die aktuell geltenden Guidelines definieren PASI75 als Therapieziel, also eine 75-prozentige Reduktion der Krankheitsaktivität vom Ausgangswert. Diese müssen aktualisiert, PASI90 als neues Therapieziel definiert werden. Viel wichtiger aber ist, dass die Guidelines auch wirklich in der klinischen Praxis auf breiter Basis umgesetzt werden und möglichst viele Patienten rasch Zugang zu innovativen Therapien erhalten. Dazu zählt ein immer breiteres Therapiespektrum mit so genannten "small molecules" und zielgerichteten Therapien, die ein immer schnelleres Ansprechen und eine immer effektivere Reduktion wichtiger Krankheitssymptome ermöglichen. Ziel ist, dem Patienten eine sichere und wirksame Langzeittherapie bei weitgehender Erscheinungsfreiheit zu ermöglichen - und so die Beeinträchtigung der Lebensqualität durch die Erkrankung zu minimieren."

hautnah $2015 \cdot 14: 75$

DOI 10.1007/s12326-015-0172-2

(c) Springer-Verlag Wien 2015

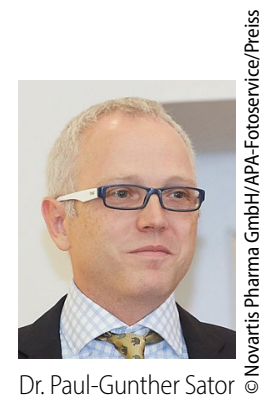

Quelle: Pressegespräch "Schuppenflechte: Ist Erscheinungsfreiheit ein realistisches Therapieziel?", Wien, 2. Oktober 2015 